\title{
The phytogestrogenic stilbenes, arachidin-1 and resveratrol, modulate regulatory $T$ cell functions responsible for successful aging in aged ICR mice
}

\author{
BRIAN BOR-CHUN WENG ${ }^{1}$, WEN-SHIN LIN ${ }^{1}$, JU-CHUN CHANG ${ }^{2}$ and ROBIN YIH-YUAN CHIOU ${ }^{2}$ \\ Departments of ${ }^{1}$ Microbiology, Immunology and Biopharmaceuticals, and ${ }^{2}$ Department of Food Science, \\ College of Life Sciences, National Chiayi University, Chiayi, Taiwan, R.O.C.
}

Received December 11, 2015; Accepted October 10, 2016

DOI: $10.3892 /$ ijmm.2016.2792

\begin{abstract}
CD}^{+} \mathrm{CD} 25^{+}$regulatory $\mathrm{T}$ cells (Tregs) are recognized as a distinctive $\mathrm{T}$ helper cell population which controls immunosuppression during the maintenance of immunological self-tolerance and immunohomeostasis. Sex steroids modulate fundamental immune functions, including immune cell development, differentiation and polarization, and facilitate specific immunophysiological microenvironments, such as pregnancy. The supplementation of exogenous phytoestrogens is beneficial to post-menopausal women. Stilbenes are a potent group of phytoestrogens, of which resveratrol (Res) is a well-known representative exhibiting a variety of immunomodulatory activities, including the attenuation of autoimmune diseases and boosting anti-tumor immunity. In the present study, arachidin-1 (Ara-1) and Res, primary stilbenes, enriched in peanut sprouts as phytoalexins, were investigated for their immunomodulatory properties for successful aging. We found that similar to $17-\beta$-estradiol (E2), Ara-1 or Res significantly inhibited concanavalin A (ConA)-activated lymphoblastogenesis of cell repertories from splenic or thymic origins. However, these inhibitory effects were partially reversed by the E2 receptor blocker, tamoxifen. While the ratios of the $\mathrm{CD} 4{ }^{+} \mathrm{CD} 25^{+}$cell population of ConA-activated $\mathrm{T}$ cell repertories were not significantly altered, treatment with E2, Ara-1 or
\end{abstract}

Correspondence to: Professor Brian Bor-Chun Weng, Department of Microbiology, Immunology and Biopharmaceutical Sciences, College of Life Sciences, National Chiayi University, 300 University Road, Chiayi 600, Taiwan, R.O.C.

E-mail: brian@mail.ncyu.edu.tw

Professor Robin Yih-Yuan Chiou, Department of Food Science, College of Life Sciences, National Chiayi University, 300 University Road, Chiayi 600, Taiwan, R.O.C.

E-mail: rychiou@mail.ncyu.edu.tw

Key words: phytoestrogen, arachidin-1, resveratrol, regulatory T cells, nuclear transcription factor forkhead box P3, cytotoxic T lymphocyteassociated antigen 4 , aging
Res led to an increase in the number of cytotoxic T-lymphocyte associated protein 4 (CTLA-4; also known as CD152)-positive cells and in the gene expression levels of CTLA-4, Forkhead box P3 (FoxP3), interleukin (IL)-10 and transforming growth factor- $\beta$ (TGF- $\beta$ ). When low (L-S-PNT) and high (H-S-PNT) levels of stilbene-enriched peanut sprout-fortified diets were provided ad libitum to 12-week-old ICR mice for 48 weeks, their circulating Treg populations were assessed following magnetic bead enrichment. The gene expression levels of CTLA-4 and TGF- $\beta$ were significantly $(\mathrm{P}<0.05)$ elevated, as assessed by semi-quantitative RT-PCR. The findings of the present study support the beneficial roles of the phytoestrogenic stilbenes, Res and Ara-1, in facilitating a successful aging immune status which may attribute to longevity.

\section{Introduction}

The optimization of immune functions is a lifelong task for an individual's well-being and is possibly the ultimate solution for longevity in addition to an individual's genetic contribution as regards lifespan. There is evidence to indicate that a group of suppressive $\mathrm{T}$ cells $\left[\mathrm{CD} 4^{+} \mathrm{CD} 25^{+}\right.$Forkhead box P3 (FoxP3) ${ }^{+}$ $\mathrm{T}$ cells, namely natural regulatory $\mathrm{T}$ cells (Tregs); and $\mathrm{CD} 4^{+} \mathrm{CD} 25^{+}$interleukin (IL) $-10^{+}, \mathrm{CD} 4^{+} \mathrm{CD} 25^{+}$transforming growth factor (TGF)- $\beta^{+}$T cells, namely inductive Tregs] is critical in maintaining immunohomeostasis in elderly subjects $(1,2)$. During advanced aging, autoantigens are frequently generated from aged organs and tissues, caused by the decline in the antioxidant capacity to scavenge harmful free radicals. Progressive pro-inflammatory conditions or related disease symptoms often have severe health consequences to aged individuals. To compensate the overwhelming oxidative stress and inflammation, the immune system undergoes a profound adaptation by diverting its $\mathrm{T}$ effector cell responses toward $\mathrm{CD}^{+} \mathrm{CD} 25^{+}$Treg-mediated immunity. The Treg population increases with age and is significantly higher in centenarians $(1,3)$. Naturally, Tregs composed $5-10 \%$ of CD4 $\mathrm{T}$ helper cell populations possess immunosuppressive functions by secreting surface CD25, cytotoxic T lymphocyteassociated antigen 4(CTLA-4; CD152), glucocorticoid-induced tumor necrosis factor receptor (GITR) and also producing anti-inflammatory cytokine, such as IL-10 and TGF- $\beta(4,5)$. 
The specific nuclear transcription factor, FoxP3, is tightly associated with Treg development and regulatory functions. Tregs can be further divided into FoxP3-expressing natural Tregs developed in the thymus, and peripheral-induced Tregs which express low levels of FoxP3, but mainly generate IL-10 or TGF- $\beta(6,7)$.

Estrogen synthesized from cholesterol through steroidogenesis with no gender bias tightly modulates immune cells of both myeloid and lymphoid origin, thus assisting reproductive immune tolerance. Estrogen controls the production of proinflammatory mediators, chemokines and chemokine receptor expression, as well as the regulation of TNF- $\alpha$ and inducible nitric oxide synthase(iNOS) expression $(8,9)$, suggesting that estrogen has an anti-inflammatory property. Indeed, in pregnancy, high levels of estrogen and progesterone levels have been shown to have ameliorative effects on autoimmune diseases, whereas pathogenic inflammatory conditions may be exacerbated during the menstrual cycle due to the decline in estrogen levels. Moreover, post-menopausal women deficient in estrogen, or estrogen receptor (ER) knockout or ovariectomized animals are all prone to developing autoimmune-related diseases $(10,11)$. Although discrepancies in the lymphoid tissue distributions of the ER $\alpha$ and $\beta$ isoforms still exist in different animal species, the ER is expressed in the thymus and peripheral blood mononuclear cells in response to fluctuating serum estradiol levels during the estrous cycle.

Due to structural similarities, stilbenes exhibit phytoestrogenic activities. Among the family of stilbenoids, resveratrol (Res; trans-3,5,4'-trihydroxystilbene) is most wellstudied for its biological activities. The phytoestrogenic activity of Res has been well documented and discussed (12-14). Res is abundantly found in grapes, blueberries, peanuts and in the TCM herb Rhizoma polygoni Cuspidati (15). Stilbenes are secondary metabolites produced in peanuts during infection with Aspergillus flavus (16), and stilbenes, including Res, isopentadienylresveratrol, arachidin-1 (Ara-1) and arachidin-3 are available through the enhanced biosynthesis of floating peanut sprout cultivation (17). Res is able to compete with estrogen on ER binding (18) and activates the ER-mediated phosphoinositide-3 kinase signaling pathway (19). Ara-1 of the major peanut stilbenoids possesses anti-inflammatory activity by inhibiting lipopolysaccharide (LPS)-induced iNOS activity, CCAAT-enhancer-binding protein $(\mathrm{C} / \mathrm{EBP}) \delta$ expression and nuclear factor- $\mathrm{\kappa B}(\mathrm{NF}-\kappa \mathrm{B})$ activation in macrophages (20). Gao et al (21) reported that Res suppressed cell-mediated cytotoxicity, interferon- $\gamma$ (IFN- $\gamma$ ) and IL-2 production, as well as concanavalin $\mathrm{A}$ (ConA)-induced lymphocyte proliferation through the nhibition of NF- $\mathrm{KB}$ activation.

Immunomodulatory evidence is still lacking to demonstrate that the stilbenes, Res and Ara-1, may have similar effects to those of estrogen by altering the Treg ratio and functional activities. Moreover, the question of whether Ara-1, a major stilbene derived from peanut sprouts extract would have better biological activity than Res in immunomodulation remains to be determined. In order to shed light on this matter, in the present study, the immunomodulatory activities of the stilbenes, Res and Ara-1, were assessed. The mechanisms associated with their phytoestrogenic properties in modulating aging-associated $\mathrm{CD} 4^{+} \mathrm{CD} 25^{+}$Treg functions were examined in both in vitro and in vivo experiments.

\section{Materials and methods}

Animals and immune cell culture. Forty healthy female Balb/c mice aged 6-8 weeks were purchased from the National Laboratory Animal Center and all animal experimentations met the regulations of the Institution of Animal Care and Utilization Committee, National Chiayi University, Chiayi, Taiwan. The animals were raised on standard laboratory rodent chow (LabDiet, Richmond, VA, USA) were euthanized by an overdose of $\mathrm{CO}_{2}$, and splenocytes were subsequently harvested by the Ficoll Histopaque (Sigma Co., Ltd., St. Louis, MO, USA) gradient separation technique as previously described by Weng et al (22). Spleno-lymphocytes suspended in $10 \%$ fetal bovine serum (FBS) with RPMI-1640 medium (phenol red free) (Sigma Co., Ltd.) were automatically counted to determine the number of live or dead cells by Trypan blue exclusion assay (Cellometer ${ }^{\circledR}$ Auto T4; Nexcelom Bioscience, Lawrence, MA, USA) and adjusted live $5 \times 10^{5}$ cells were then seeded onto 96-well plates for further assays. All materials, unless otherwise indicated, were purchased from Sigma Co., Ltd. Plastic ware was obtained from Corning Co., Ltd. (Corning, NY, USA). Cell cultures were performed in a humidified, $37^{\circ} \mathrm{C}$, $5 \% \mathrm{CO}_{2}$ incubator.

Cytotoxicity of stilbenes to lymphocytes. MTT assay was used to determine lymphocyte viability following incubation with Res or Ara-1 [Res and Ara-1 were biosynthesized and purified by floating peanut sprout cultivation in our laboratory previously described by Chang et al (17). A commercial Res was purchased and used as standard for comparative checking purposes]. Res and Ara-1 (1, 5, 10 and $25 \mu \mathrm{M})$ were prepared by dissolving with dimethyl sulfoxide (DMSO). The final concentration of DMSO was controlled under $0.1 \%(\mathrm{v} / \mathrm{v})$. MTT solution $(0.5 \mathrm{mg} / \mathrm{ml})$ was added to each well followed by incubation for $4 \mathrm{~h}$. The MTT formazan is reduced to purplish formazan crystals, indicating proliferating cells. This was then dissolved by the addition of a $95 \% \mathrm{EtOH}$ and DMSO mixture. The absorbance was measured at $\mathrm{OD}=570 \mathrm{~nm}$ using an ELISA reader (Biotek Instruments, Inc., Winooski, VT, USA).

ConA-induced lymphocyte proliferation assay. The $\mathrm{T}$ cell mitogen, ConA, was used to non-specifically activate $\mathrm{T}$ cell polyclonal proliferations at a predetermined sub-optimal concentration of $5 \mu \mathrm{g} / \mathrm{ml}$. An aliquot of $100 \mu 1,5 \times 10^{5}$ cells purified lymphocytes from either splenocytes or thymocytes was seeded into 96-well round-bottom plates of a phenol-red free basal medium in the presence of estrogenic treatments for $24 \mathrm{~h}$ prior to mitogen stimulation. The cells were pre-incubated with either E2 (17- $\beta$-estradiol, 160 pM; Cat. no. 3301; Merck, Darmstadt, Germany), Res at concentrations of 5, 10 and $25 \mu \mathrm{M}$, or Ara- 1 at concentrations of $0.5,1$ and $5 \mu \mathrm{M}$, and an additional $20 \mu \mathrm{l}$ ConA was then added for an extended $48 \mathrm{~h}$ of incubation at $37^{\circ} \mathrm{C}, 5 \% \mathrm{CO}_{2}$ in a humidified incubator. During the incubation period, $20 \mu 1$ Alamar Blue Dye ${ }^{\mathrm{TM}}$ (Accumed International Inc., Chicago, IL, USA) were added to each well $24 \mathrm{~h}$ before the end of incubation. The dye is in a blue oxidized form (resazurin) and is reduced to resorufin with a red color when cells are proliferating. The level of lymphocyte proliferation was determined by the change in absorbency when the fluorescent dye was reduced. The difference between the mean 
values of absorbence of duplicates from the unstimulated cell medium and the ConA-stimulated cell medium was detected using a microplate reader (FLx800; BioTek, Winooski, VT, USA) to measure the reduced form at an excitation of $528 \mathrm{~nm}$ and the oxidized form at an emission of $590 \mathrm{~nm}$, as previously described (22). The percentage proliferation index was calculated as follows: the value of the treatment group divided by that of the control group.

Cytokine concentrations in culture supernatants. The TGF- $\beta$, and IL-10 levels in the supernatant of ConA-activated lymphocyte repertories of spleno-lymphocytes or thymocytes were assessed after $48 \mathrm{~h}$ of culture. The supernatants from each assay were stored in $-80^{\circ} \mathrm{C}$ freezer until final analysis. Specific cytokines were detected by standard enzyme-linked immunosorbent assay (ELISA) using assay techniques as described in the manufacturer kit instructions (TGF- $\beta$, Cat. no. DY1679; R\&D Systems Inc., USA; and IL-10, Ready-Set-Go kit, Cat.no. 88-7904; eBioscience, San Diego, CA, USA). Duplicates of each sample were measured for their cytokine production using a microplate ELISA reader.

Lymphocyte phenotyping. ConA-stimulated lymphocyte populations of splenocytes or thymocytes were subjected to flow cytometric analysis to determine the number of $\mathrm{CD} 25^{+} \mathrm{CD}^{+} \mathrm{T}$ cell populations. $\mathrm{CD}^{+}$cells were firstly gated as the $\mathrm{T}$ lymphocyte compartment using an anti-CD3 antibody conjugated with FITC anti-CD3 antibody conjugated with FITC (Cat. no. 561798). Anti-CD4-conjugated PE antibody (Cat. no. 558642) and anti-CD25-conjugated PerCP-Cy5.5 antibody (Cat. no. 551071) (all from BD Pharmingen, San Diego, CA, USA) were used to stain the cells at $4^{\circ} \mathrm{C}$ for $45 \mathrm{~min}$ followed by washing with fresh phosphate-buffered saline (PBS) with sodium azide. Following forward and side scatter adjustment on a flow cytometer (FACScan; Becton-Dickinson, San Jose, CA, USA), the fluorescence and frequency of FL-1a, FL-2 and FL-3 channels were examined for the pan CD3 population and two color analysis of CD $25^{+} \mathrm{CD} 4^{+} \mathrm{T}$ cells. Values are presented as the mean percentage of the total cell population of 3 independent experiments. Three-color fluorescence flow cytometric analysis was performed to determine CTLA-4 (anti-CD152 conjugated FITC antibody; BD Pharmingen) expression. Logical gated FL-2- and FL-3-positive populations were then analyzed for CTLA-4 expression (FL-1). Data were analyzed using WinMDI ${ }^{\circledR}$ (Free software, Flow Cytometry Core Facility, Scripps Institute, La Jolla, CA, USA).

Semi-quantitative determination of specific mRNA expression by $R T-P C R$. For RNA extraction, $1 \times 10^{6}$ cells were collected at designated experimental phases. Total RNA was extracted using TRIzol reagent following the manufacturer's instructions (Protech Technology Enterprise Co., Ltd, Taipei, Taiwan). RNA was reverse transcribed using a cDNA synthesis kit (Promega, Madison, WI, USA). Synthesized cDNA was then amplified with Taq polymerase and specific primers for $\beta$-actin, FoxP3, CTLA- 4 and TGF- $\beta$ in a thermal cycler. The optimum conditions for RT-PCR were $58^{\circ} \mathrm{C}$ for $0.5 \mathrm{~min}, 60^{\circ} \mathrm{C}$ for $0.5 \mathrm{~min}$, and $55^{\circ} \mathrm{C}$ for $0.5 \mathrm{~min}$ for 30 cycles for $\beta$-actin, 30 cycles for FoxP3, 35 cycles for CTLA-4, and 30 cycles for TGF- $\beta$, respectively, and a final extension at $72^{\circ} \mathrm{C}$ for $7 \mathrm{~min}$.
The primers used in this study and the product size were 5'-TCTACGAGGGCTATGCTCTCC-3' (sense) and 5'-GGATGCCACAGGATTCCATAC-3' (antisense) for $\beta$-actin (340 bp); 5'-TTCACCTATGCCACCCTTATCC-3' (sense) and 5'-GGCTCCTCTTCTTGCGAAACTC-3' (antisense) for FoxP3 (216 bp); 5'-ATTCACCATCACACAACACT-3' (sense) and 5'-GGGGCATTTTCACATAGACC-3' (antisense) for CTLA-4 (483 bp); 5'-CTGTCCAAACTAAGGCTCGC-3' (sense) and 5'-CGTCAAAAGACAGCCACTCA-3' (antisense) for TGF- $\beta$ (440 bp). A total of $25 \mu \mathrm{l}$ of final PCR products was analyzed by electrophoresis on $1.2 \%$ agarose gel in TBE buffer. The bands were visualized using ethidium bromide and each band was measured and analyzed using Gel-Pro Analyzer ${ }^{\circledR}$ software (Media Cybernetics, Silver Spring, MD, USA).

Effect of estrogen receptor blocker on the stilbene-mediated inhibition of lymphocyte proliferation. 4-Hydroxytamoxifen (TAM, Cat. no. H7904; Sigma Co., Ltd.) is a known ER $\alpha$ antagonist which mimics endogenous estrogen. The cells were pre-incubated with $1 \mu \mathrm{M}$ TAM and then subjected to co-incubation with the stilbenes for $24 \mathrm{~h}$. Subsequently, Con A was added for an additional $48 \mathrm{~h}$ of incubation prior to the measurement of lymphocyte proliferation. The same methodology was used as described above in the lymphocyte proliferation assay.

Dietary intervention with a stilbene-fortified diet on sorted Treg functions in aged ICR mice. Stilbenes were extracted and purified from peanut sprouts as previously described by Lin et al (23) and Huang et al (24) and fortified into a rodent basal diet at levels of $15 \mathrm{mg}$ (L-S-PNT; low) and $75 \mathrm{mg}$ (H-SPNT; high) per kilogram body weight per day. Peanut sprout powder deficient in stilbenes following extraction was used as a vehicle control (PNT) at the same quantity as the treatment groups. The control group (negative control, NC) was fed basal rodent diet only. Animals were purchased from the National Laboratory Animal Center, Taiwan, and all animal experimentations met the regulations of the Institution of Animal Care and Utilization Committee, National Chiayi University, Chiayi, Taiwan. A total of 40 12-week-old male ICR mice were equally assigned into 4 dietary treatment groups as follows: the control group, the PNT group, the L-S-PNT group and the H-S-PNT group. The mice were fed on an libitum basis for an entire 48 weeks until they were euthanized by an overdose of $\mathrm{CO}_{2}$ to determine the ratio of Tregs. For monitoring purposes, measurements of body weight and blood biochemicals, and hematological analysis were performed, and the results did not reveal any statistically significant differences among the treatment groups (data not shown). Spleno-lymphocyte Treg subset analysis was performed as described above. The spleens of 3 mice from each group were aseptically removed and forced through a stainless steel wire mesh. Spleno-lymphocytes were then harvested by Ficoll gradient separation. The splenolymphocytes of 3 mice were pooled for $\mathrm{CD} 4{ }^{+} \mathrm{CD} 25^{+}$Treg enrichment by magnetic bead separation. The $\mathrm{CD} 4^{+} \mathrm{CD} 25^{+}$ Treg cell enrichment protocol was performed according to the manufacturer's recommendations (regulatory $\mathrm{T}$ cell isolation kit, Cat. no. 130-091-041; Miltenyi Biotec Inc., Bergisch Gladbach, Germany). Briefly, non-CD $4^{+}$cells were removed magnetically with a cocktail of biotin-labeled antibodies and 
anti-biotin microbeads using a large column. Subsequently, CD25-positive selection was performed using specific microbead-labeled antibody using a small column. Enriched Tregs were then subjected to RNA extraction for the determination of CTLA-4 and FoxP3 expression as described above.

Statistical analysis. All data were conducted using the GLM model procedure (SAS Institute, 1996) for statistical analysis. Significant differences among the treatment groups were determined using Fisher's LSD test. Values represented in the bar graphs are the means \pm standard deviation (SD). For the in vitro experiments, the Student's t-test was used for paired comparisons among experimental groups.

\section{Results and Discussion}

Immunotoxicity of stilbenes and their inhibitory effects on ConA-induced lymphoblastogenesis. The silbenes, Res or Ara-1, extracted from peanut sprout powder were purified $(17,24)$ and assessed for their cytotoxicity to murine lymphocytes (Fig. 1). The IC50 value of Ara-1 was approximately $25 \mu \mathrm{M}$, while little or no toxicity was observed with Res at the same concentration. Ara-1 has a chemical structure similar to Res, but an additional hydroxyl and isopentenyl moiety, which possibly facilitates a better biological potency. It is not chemically synthesized and has been shown to inhibit LPS-induced nitric oxide production in murine macrophages by reducing the expression of C/EBP $\delta$ and NF- $\kappa B$ signaling at concentrations $<15 \mu \mathrm{M}(20)$. Hence, the concentrations of Res and Ara-1 used in the ex vivo experiments were maximum at 25 and $5 \mu \mathrm{M}$, respectively.

To reduce progressive $\mathrm{T}$ cell responses to aging-associated self-antigens, Tregs are largely expanded in successfully aged individuals to offset the pathogenic autoimmune diseases resulting from overreactive $T$ cells. The non-specific polyclonal activation of $\mathrm{T}$ cell blastogenesis by ConA was setup as a 72-h culture period with an initial $24 \mathrm{~h}$ of priming with different concentrations of Res, Ara-1, E2 or medium only (ConA only) prior to $48 \mathrm{~h}$ of stimulation with ConA. T cell proliferation was presented as an index of proliferation (\%) as a percentage of the proliferation of ConA-stimulated lymphocytes. Pre-culture with 160 pM E2 exerted an approximately 20 and $40 \%$ inhibitory effect on lymphoblastogenesis in the spleen and thymus respectively. All levels of Res, apart from the lowest level, exerted a significant $(\mathrm{P}<0.05)$ inhibitory effect on ConA-stimulated $\mathrm{T}$ cell proliferation. At the concentration of $25 \mu \mathrm{M}$, Res exerted a similar effect as E2, and the inhibitory effects were more prominent on the lymphocytes from the thymus than those from the spleen (Fig. 2A). Similar to the inhibitory effects of E2 and Res, Ara-1 exerted a more prominent $(\mathrm{P}<0.001)$ inhibitory effect on lymphoblastogenesis at the concentration of $5 \mu \mathrm{M}$, whereas Res did not affect ConA-stimulated lymphoblastogenesis at the same concentration. In general, the inhibitory effects were more prominent on the thymus lymphocytes in all estrogenic treatments. Both stilbenes did not exert any cytotoxic effects on the lymphocytes at the concentration range tested in the present experiment; thus, the inhibition of cell proliferation due to cytotoxicity was devoid. It has been reported that E2 and diethylstilbestrol (DES) exert suppressive modulatory effects on lymphocyte activation and blastogenic responses,

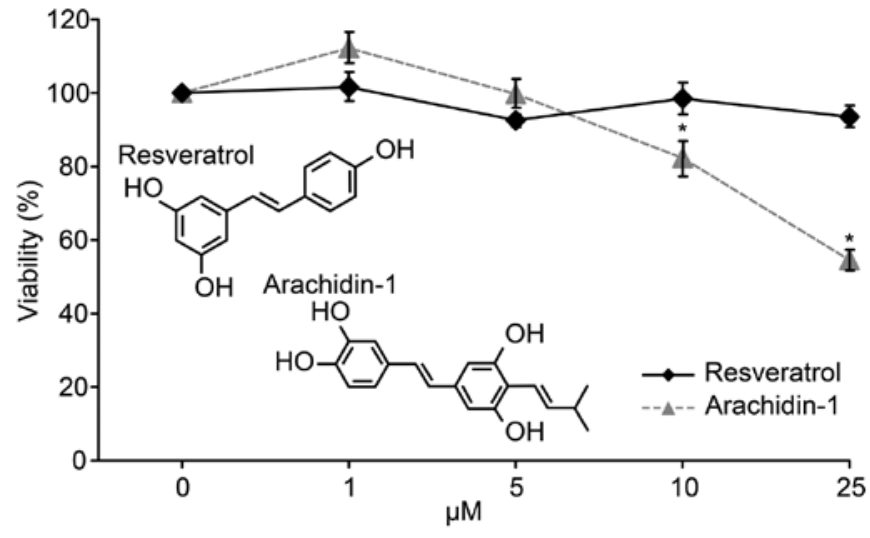

Figure 1. The cytotoxicity of resveratrol and arachidin-1 of peanut sprout extracts in spleno-lymphocytes was assessed with MTT assay. Arachindin-1 had higher toxicity than resveratrol and significantly reduced lymphocyte viability at concentration higher than $10 \mu \mathrm{M}$ as compared to that of resveratrol. At concentration up to $25 \mu \mathrm{M}$, resveratrol showed negligible cytotoxicity in spleno-lymphocyte culture. Data shown were mean \pm SD of three independent assays.

and alter the peripheral $\mathrm{T}$ cell repertoire by altering thymic selection $(25,26)$. In addition, a previous study using $20 \mu \mathrm{M}$ Res demonstrated that ConA-induced lymphocyte proliferation was suppressed (21), which may be attributed to the decreased expression of the T cell co-stimulatory molecules, CD28, and CD80 of antigen-presenting cells $(3,21)$. CTLA- 4 is known to compete with $\mathrm{CD} 28$ in their ligands and mediates immunosuppression that is often observed with upregulated regulatory T cell activity (31). Whether CTLA-4 expression causes the unresponsiveness to ConA-induced lymphoblastogenesis shall be firstly tested if the inhibitory efffects of stilbenes on ConAactivated lymphoblastogenesis is attributed to their estrogenic properties.

Recovery of stilbene-mediated suppression of lymphoblastogenesis by tamoxifen. Mixed lymphocyte reaction (MLR) resembles the lymphocyte proliferation reaction and is downregulated by elevated estrogen concentrations, and is known to be essential in minimizing allograft rejection for successful pregnancy. Res acts as a phytoestrogen, exerting agonistic effects with ER in lymphocytes to modulate mitogen-induced $\mathrm{T}$ cell proliferation (21). Similarly, our results demonstrated that the ConA-induced lymphoblastogenesis of spleno- or thymic lymphocytes was suppressed by Res and Ara-1 in a dose-dependent manner, and the inhibitory effects were more promiment with Ara- 1 at a concentration range of 0.5 to $5 \mu \mathrm{M}$ (Fig. 2A). When the lymphocytes were pre-incubated with tamoxifen, a known ER $\alpha$ blocker, the suppressive effects of Res and Ara-1 on ConA-induced lymphoblastogenesis were attenuated, and lymphoblastogenesis returned to levels similar to those of the ConA only group treated with ConA alone (Fig. 2B). Moreover, partial recovery was observed in the thymic lymphocytes treated with high levels of Res $(25 \mu \mathrm{M})$ or Ara-1 $(5 \mu \mathrm{M})$, which indicated that the thymus is more prone to estrogenic modulation. The recovery of lymphoblastogenesis by pre-treatment with tamoxifen indicated that the immunosuppressive effects of the stilbenes was similar to those of E2 and that they are mediated through the ER-mediated cellular signaling pathway. ER has two isoforms, ER $\alpha$ and $E R \beta$, which 

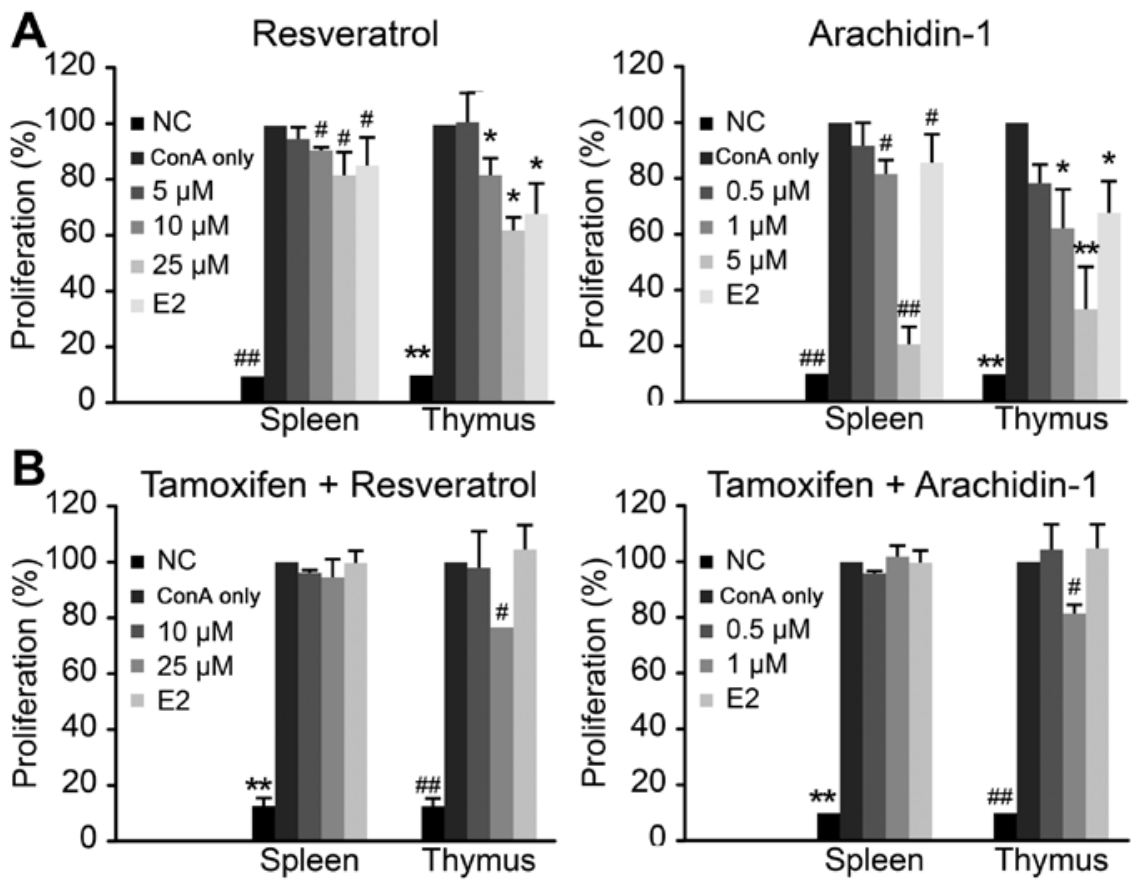

Figure 2. The dose-dependent inhibition of resveratrol and arachidin-1 on concanavalin A (ConA) activated lymphoblastogenesis of spleno- and thymic lymphocytes are shown (A). The highest level of arachidin-1 was 5 times less than resveratrol which implies a higher potency of arachidin-1. The suppressive effects of arachindin-1 and resveratrol were similar to that of estradiol (E2) at a physiologically relative concentration of 160 pM. When lymphocytes were pretreated with $1 \mu \mathrm{M}$ tamoxifen (ER blocker), partial recovery on the inhibition of ConA-activated lymphoblastogenesis of E2, resveratrol or arachidin-1 was demonstrated (B). The immunosuppression mediated by arachidin-1 and resveratrol was similar to E2 through estrogen receptor signaling. Significant difference in comparison with the ConA only group at ${ }^{\#} \mathrm{P}<0.05$ and ${ }^{\# \#} \mathrm{P}<0.001$ of spleno-lymphocytes; significant difference in comparison with the Con $\mathrm{A}$ only group at ${ }^{*} \mathrm{P}<0.05$ and ${ }^{* *} \mathrm{P}<0.001$ of thymic lymphocytes. Data were mean $\pm \mathrm{SD}$ of three independent experiments.

are differentially distributed in various tissues. In the thymus, $\mathrm{ER} \beta$ expression is high and that of $\mathrm{ER} \alpha$ is low (28). Res binds to $\operatorname{ER} \alpha$ and $\operatorname{ER} \beta$ with similar affinity. In a previous study, at concentrations of $100 \mu \mathrm{M}$, Res was shown to exert an inhibitory effect on the proliferation of $\mathrm{CHO}-\mathrm{K} 1$ cells transfected with either ER $\alpha$ or ER $\beta$ (12). Moreover, tamoxifen and the non-steroidal estrogen, DES, are known to be ligands exclusive to the $\mathrm{ER} \alpha$ receptor containing the $\mathrm{C}$-terminal ligand binding domain (27). In immune organs, the spleen and thymus have higher a mRNA expression of ER $\beta$ than ER $\alpha$ (28); however, epithelial cells of these tissues primarily express ER $\beta$, whereas $\mathrm{ER} \alpha$ is mainly expressed in CD4 and CD8 T cells (29). In the present study, pre-incubation with tamoxifen effectively repressed the subsequent stilbene modulation of $\mathrm{T}$ lymphocyte functions. Additional pathways through other targeted receptors or cellular protein molecules of Res (those that are beyond ER-mediated activities), such as the inhibition of tyrosine kinase in cell signal transduction occur particularly during high concentration of stilbenes (13) should also be considered.

Stilbenes do not affect the $C D 4^{+} C D 25^{+}$regulatory $T$ cell population in the ConA-activated Tlymphocyte repertoire. It has been reported that the expansion of Tregs during the late menstrual cycle is tightly correlated with plasma estradiol levels (30). To determine whether phytoestrogenic stilbenes alter the Treg ratio, we analyzed the repertoire of Tregs in the ConA-stimulated thymic or spleno- lymphocytes pre-treated with E2, Res or Ara-1. Flow cytometry detected an approximately $10 \%$ increase in the $\mathrm{CD} 4^{+} \mathrm{CD} 25^{+} \mathrm{T}$ cell population following stimulation with ConA (ConA only group) when compared with the unstimulated lymphocyte population (data not shown). Following stimulation with ConA, the CD25+ $\mathrm{T}$ cell population was markedly increased (data not shown). Anti-CD25 antibody is specific for capturing the IL-2 $\alpha$ subunit. IL-2 receptor expression increases in proliferating T cells in an IL-2 autocrine manner (6). In this study, to obtain stably expanded $\mathrm{T}$ cell colonies at a resting state, samples were replaced with fresh medium for a 12-h extention of culture prior to flow cytometric analysis. As shwon in Fig. 3, no significant changes were observed in the $\mathrm{CD} 4{ }^{+} \mathrm{CD} 25^{+} \mathrm{T}$ cell ratio among the E2-, Res- or Ara-1-treated cells as compared to the ConA only group (ConA-activated lymphoblast repertoire of either spleno- or thymic lymphocytes). It has been suggested that $\mathrm{CD}^{+}{ }^{+} \mathrm{CD} 25^{+}$cells remain at a fixed ratio relative to $\mathrm{CD} 4{ }^{-} \mathrm{CD} 25^{+}$cells, even though the absolute number of a particular T cell is clonally expanded (31). In addition, it has been reported that follicular or gestational high-level E2 may expand Treg compartments to maintain an immunological tolerance (30). In the present study, lymphocytes pre-treated with E2 at a physiological gestation level of 160 pM, or with Res or Ara-1 had an overall lower proliferative index than the ConA only group. This may be attributed to the inhibitory effects of estrogenic stilbenes on the $\mathrm{CD} 4{ }^{+} \mathrm{CD} 25^{+}$Treg cell populations. For better elucidation, we further characterized Treg cell functional activity by the unique nuclear transcription factor, FoxP3, and their essentially expressed inhibitory molecule, CTLA-4 as affected by phytoestrogenic stilbenes.

Functional activity of Tregs treated with stilbenes in the ConA-activated Tlymphocyte repertoire. CTLA-4 is well 

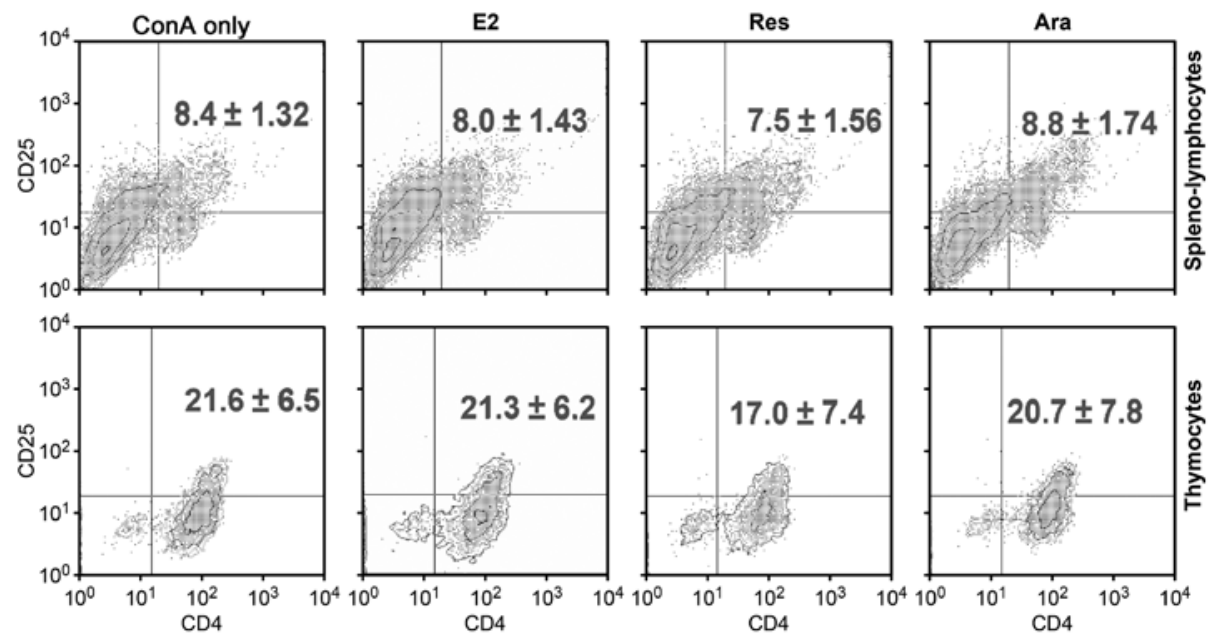

Figure 3. Analysis of $\mathrm{CD}^{+} \mathrm{CD} 25^{+} \mathrm{T}$ cells sub-population of concanavalin A (ConA) activated spleno- or thymic lymphocytes (ConA only) treated with either estrogen (E2), resveratrol (Res) or arachidin-1 (Ara) or vehicle control were demonstrated. There was no significant (P>0.05) alteration on the ratio of $\mathrm{CD} 4{ }^{+} \mathrm{CD} 25^{+} \mathrm{T}$ cells population among treatments. Data were mean $\pm \mathrm{SD}$ of at least three independent experiments.

documented as a contact inhibitory molecule expressed in Tregs. CTLA-4 expression in the $\mathrm{CD} 4{ }^{+} \mathrm{CD} 25^{+} \mathrm{T}$ cells from the ConA-stimulated lymphocytes from the E2-, Res-, or Ara-1-treated groups was examined and the results are shown in Fig. 4A. Flow cytometric histograms demonstrated that the lymphocytes pre-cultured with E2, Res or Ara-1 had an increased the mean ratio of CTLA-4-expressing populations following stimulation with ConA. It should be noted that the $\mathrm{T}$ cells analyzed in the present study were pre-cultured with E2, Res or Ara- 1 for $24 \mathrm{~h}$ prior to a $48 \mathrm{~h}$ of stimulation with ConA, followed by an additional $12 \mathrm{~h}$ of culture in fresh medium by replacing mitogen containing medium with fresh RPMI-1640 basal medium (without FBS). This is to allow mitogen-activated $\mathrm{T}$ cells to return to a resting state for better estimation of the CTLA-4-expressing population. Moreover, the semiquantitative analysis of the gene expression of CTLA-4 was also carried out and results are shown in Fig. 4B. The results of the density of CTLA- 4 mRNA expression exhibited a $>50 \%$ increase $(\mathrm{P}<0.05)$ in the Ara-1 pre-treated spleno-lymphocytes as compared to the ConA only group. Although the Res-treated cells exhibited a similar enhancement as the Ara-1-treated cells, the effective concentration of Res $(25 \mu \mathrm{M})$ was much higher than that of Ara-1 $(5 \mu \mathrm{M})$. In general, thymocytes were less responsive to stilbene modulation, while E2 exerted more prominent $(\mathrm{P}<0.05)$ promoting effects on $\mathrm{CTLA}-4$ gene expression. It has been reported that CTLA-4 gene expression protects mice from experimental autoimmune encephalomyelitis (32). CTLA-4 expression has also been shown tobe upregulated in the $\mathrm{CD} 4{ }^{+} \mathrm{CD} 25^{+}$Tregs of pregnant women (33). Using concentrations of 1 to $20 \mu \mathrm{M}$ of Res, Sharma et al (34) did not demonstrate changes in CTLA-4 expression in ConAstimulated $\mathrm{T}$ cells, but repressed lymphocyte proliferation by the downregulation of the expression of CD28 and CD80. The discrepancy in the modulatory effects of Res on CTLA-4 expression require further investigation. Ara-1 belongs to the family of stilbenoids and is structurally similar to Res, but with an additional hydroxyl group and terpene tail; it exhibited greater potency than Res in stimulating CTLA-4 expression. Moreover, we also demonstrated that FoxP3 gene activity was upregulated by E2, as well as by Res and Ara- 1 in both the ConA-activated spleno- and thymic lymphocytes (Fig. 4C). The FoxP3-transduced Tregs (natural Tregs) are found in high numbers in mice positive for estrogen (17- $\beta$-estradiol, E2), and FoxP3 is downregulated in ER $\alpha$-deficient animals (35). In this study, we demonstrated that the stilbenes, Res and Ara-1, acted in a manner similar to E2 in promoting FoxP3 activity, and this effect was more prominent in thymocytes. Natural Tregs developed in the thymus known to express FoxP3 compared to peripheral polarized Tregs, which mainly produce the cytokines, IL-10 and TGF- $\beta$. Hence, we further investigated whether the production of IL-10 and TGF- $\beta$ by Tregs is also affected by treatment with stilbenes, which may contribute to peripheral tolerance in leading to successful aging.

Ara-1 promotes the production of TGF- $\beta$ and IL-10 in the ConA-activated T lymphocyte repertoire. TGF- $\beta$ and IL-10 are anti-inflammatory cytokines and control pathogenic, as well as aging-associated inflammatory conditions. They are selectively produced by peripheral Treg subsets as distant mediators of immunosuppression. The productions of TGF- $\beta$ and IL-10 in ConA-activated spleno- and thymic lymphocytes, as affected by E2, Res, or Ara-1 is shown in Fig. 5A. TGF- $\beta$ production by the ConA-stimulated spleno-lymphocytes was significantly $(\mathrm{P}<0.05)$ elevated following treatment with E2 $(160 \mathrm{pM})$ or Res $(25 \mu \mathrm{M})$, but not with Ara-1 $(5 \mu \mathrm{M})$. On the other hand, Ara-1 significantly stimulated IL-10 production in both the ConA-activated spleno- and thymic lymphocytes as compared to treatment with E2, Res and ConA alone. In the ConA-activated thymic lymphocytes, IL-10 was upregulated in an order of significance which was Ara-1 $>$ Res $>$ E2 $>$ ConA only treatment. The effects of E2 and the stilbenes on TGF- $\beta$ and IL-10 mRNA levels were similar, and were exerted in an elevating manner (Fig 5B). Previous findings have indicated that physiological levels of estrogen can induce the expansion of $\mathrm{CD}^{+}{ }^{+} \mathrm{CD} 25^{+}$Tregs and the upregulation of FoxP3 and IL-10 genes expression in in vitro setting, which is attributed to the suppression of $\mathrm{T}$ cell proliferation in a mixed lymphocyte reaction (36). Our result was in agreement with that study, in 

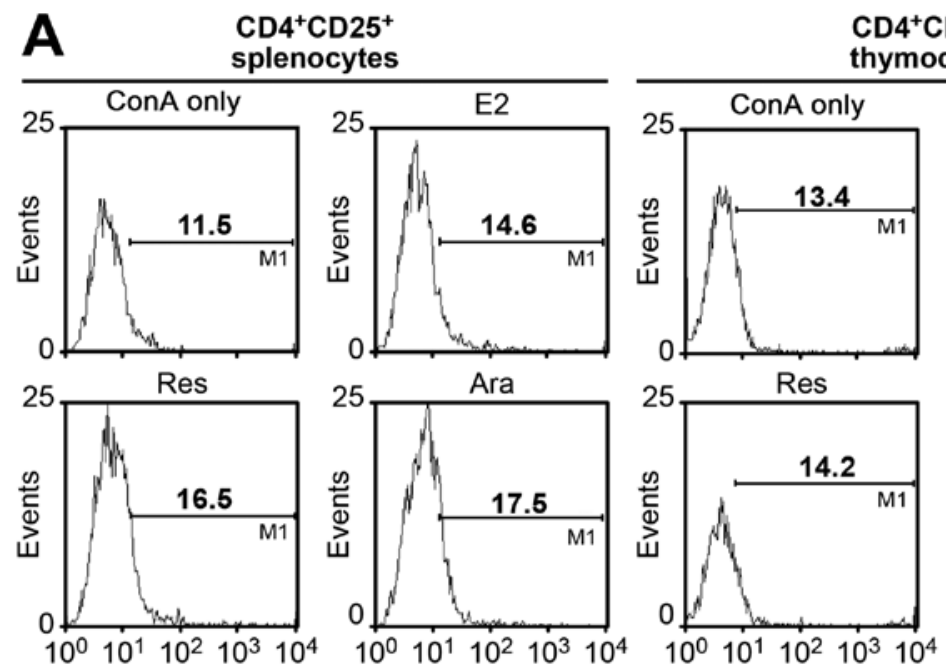

CD4 ${ }^{+}$CD25 $5^{+}$

thymocytes
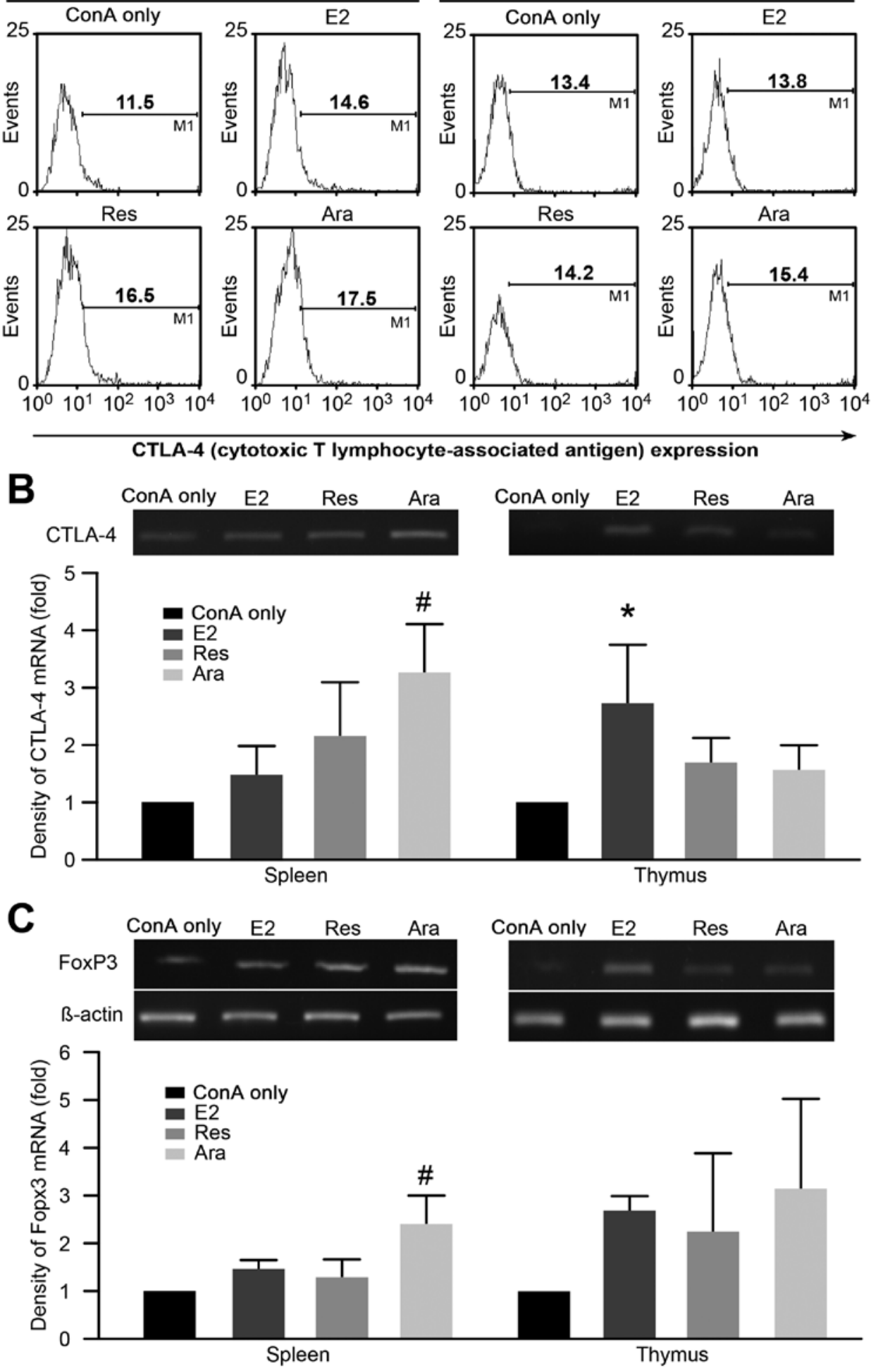

Figure 4. Flow cytometric analysis of CTLA-4 expressed on cell population of pan CD4 ${ }^{+} \mathrm{CD} 25^{+} \mathrm{T}$ cells has confirmed the resveratrol (Res) $(25 \mu \mathrm{M})$ and arachidin-1 (Ara) $(5 \mu \mathrm{M})$ were similar to the effects of E2 moderately increased the CTLA-4 expression of both spleno- and thymic CD4 ${ }^{+} \mathrm{CD} 25^{+}$lymphocytes (A). The semi-quantify gene expression of CTLA-4 confirmed that E2, Res and Ara had enhanced its expression. Moreover, Ara significantly enhanced spleno-lymphocyte CTLA-4 mRNA expression ( $\left.{ }^{*} \mathrm{P}<0.05\right)$, while E2 exhibited a stronger modulatory effect on thymic lymphocytes ( $\left.{ }^{*} \mathrm{P}<0.05\right)(\mathrm{B})$. Moreover, Forkhead box P3 (FoxP3) mRNA expression was also semi-quantify analyzed. Again, Ara treated concanavalin A (ConA)-activated spleno-lymphocytes had a significant $\left({ }^{*} \mathrm{P}<0.05\right)$ increase in FoxP3 expression as compared to that of ConA only group. The average FoxP3 mRNA expressions had an elevated-trend in thymic lymphocytes as affected by E2, Res or Ara (C). In all bar graphs, significances were treatment comparisons to respective ConA only group at $\mathrm{P}$-value $=0.05$. All data were shown in mean $\pm \mathrm{SD}$ of at least three independent experiments.

that IL-10 production was upregulated by E2; nevertheless, both phytoestrogenic Res and Ara-1 exhibited even more superior biological activity as regards IL-10 production in ConA-activated T lymphocytes. In particular, Ara-1 used at a 

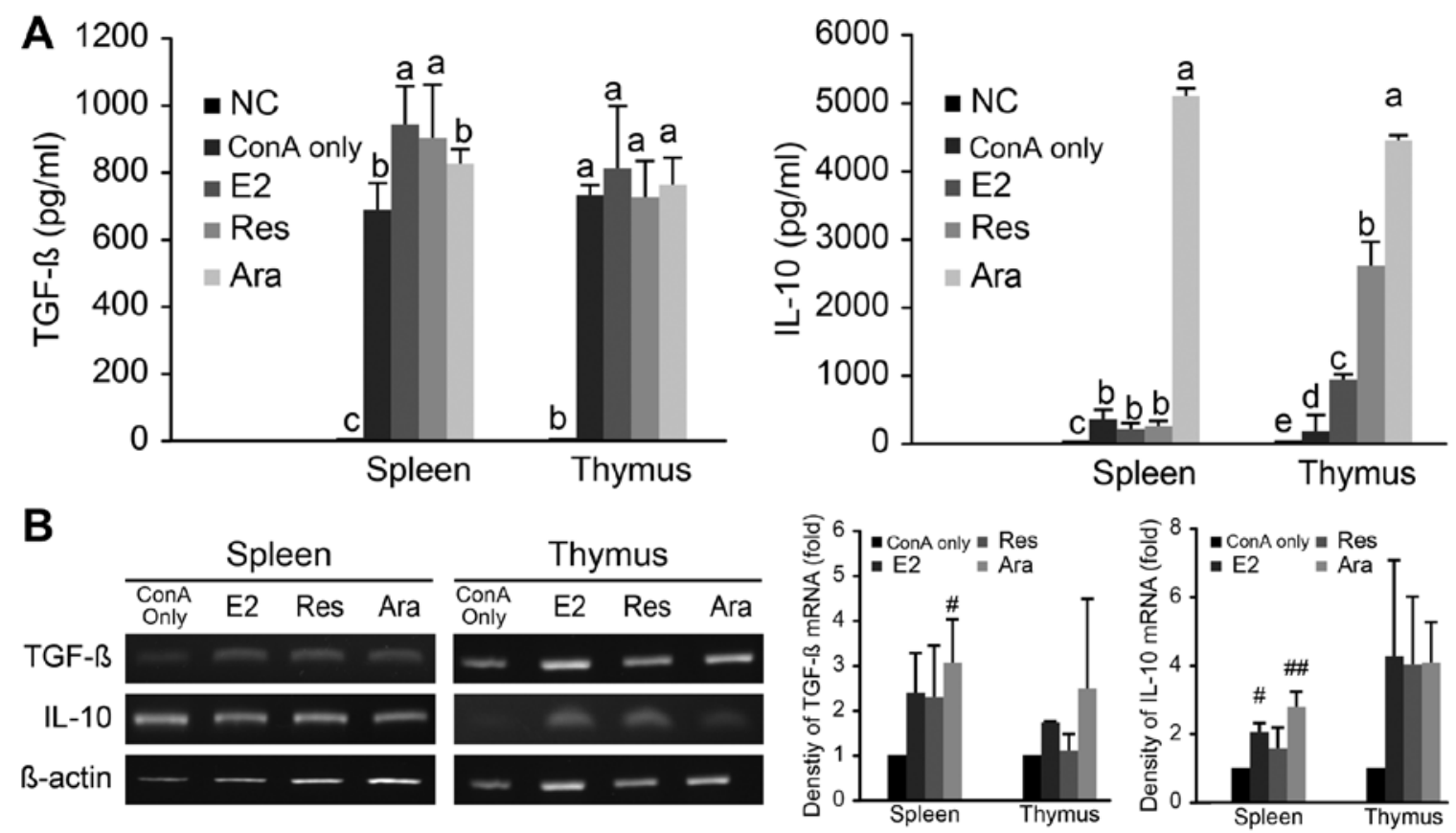

Figure 5. Productions and gene expressions of transforming growth factor- $\beta$ (TGF- $\beta$ ) and interleukin-10 (IL-10) of concanavalin A (ConA) activated spleno- or thymic lymphocytes were assessed. TGF- $\beta$ level were elevated in ConA-activated lymphocytes (ConA only) when compared with unstimulated lymphocytes (NC, negative control).. In spleno-lymphocytes, E2 and resveratrol (Res) treatments had significantly $(\mathrm{P}<0.05)$ increased TGF- $\beta$ concentration as compared with the vehicle and arachidin-1 treatments, while no significant difference among treatments of those ConA-activated thymic lymphocytes. Culture with arachidin-1 (Ara) significantly increased IL-10 production in ConA-activated lymphocytes of both spleen and thymus, whereas Res and E2 selectively affect thymocytes but only moderately affect IL-10 production of spleno-lymphocytes (A). Semi-quantitative analysis on TGF- $\beta$ and IL-10 mRNA expressions revealed that E2, Res or Ara could upregulate IL-10 and TGF- $\beta$ genes expressions of ConA-activated lymphocytes. Results of the density of specific mRNA expression as fold of house-keeping control gene ( $\beta$-actin) is also shown in bar graph (B). Data with different letters indicated significant difference between two treatment groups at $\mathrm{P}<0.05$. Significant difference in comparison with the respective ConA only group at ${ }^{\#} \mathrm{P}<0.05$ and ${ }^{\# \#} \mathrm{P}<0.001$; data were expressed as mean $\pm \mathrm{SD} ; \mathrm{n}=3$.

relatively lower concentration of $5 \mu \mathrm{M}$ than that of Res at $25 \mu \mathrm{M}$ led to a $>5$-fold greater increase in the IL-10 level. It has been reported that Res binds $E R \alpha$ and $E R \beta$ with comparable affinity, but with a 7,000-fold lower affinity than E2 (12). Whether the estrogen receptor binding affinity of Ara-1 is higher requires further investigation. Moreover, DES is a synthetic compound that is chemically similar to stilbenes. It has potent estrogenic activity via ER $\alpha$, and has been reported to alter the T cell repertoire in the peripheral blood by influencing thymic selection of T cell development (26). Taken together, Res and Ara-1 of the stilbenoid family exhibited similar properties as those of E2 in modulating the production of TGF- $\beta$ and IL-10, and their immunomodulatory effects were exerted at the mRNA level. Ara-1 seems to be more potent than Res in promoting the immunosuppressive cytokines, TGF- $\beta$ and particularly IL-10 production in lymphocytes. The phytoestrogenic properties of stilbenes hold immunological promises in complying with the successful aging phenomenon of Treg dominant immunity.

Long-term dietary supplementation of peanut sprout powder rich in stilbenes boosts Treg activity in aged ICR mice. Stilbenes are naturally abundant in a wide variety of plant species, and are produced as phytoalexin via the phenylpropanoid synthesis pathway to protect against microbial infections (15). In previous studies, we demonstrated that Res, Ara-1, arachidin-2 and archidin-3 are abundantly found in peanut sprouts and possess antioxidant properties $(15,17)$. In this study, dietary supplementation experiments, by providing either a basal diet, peanut sprout only (PNT) diet or a peanut sprout diet fortified with additional $15 \mathrm{mg} / \mathrm{kg}$ body weight /day (L-S-PNT) or $75 \mathrm{mg} / \mathrm{kg}$ body weight /day (H-S-PNT) of stilbenes extracts, were conducted. Adult male ICR mice fed the experimental diets for 48 weeks were at their last trimester of a known 2 -year estimated lifespan of murine species. $\mathrm{CD} 4{ }^{+} \mathrm{CD} 25^{+}$ $\mathrm{T}$ cell subset phenotyping of lymphocytes harvested from the spleen was carried out as shown in Fig. 6A. In general, the aging mice fed either the low or high stilbene-rich diet had a similar ratio of the $\mathrm{CD} 4{ }^{+} \mathrm{CD} 25^{+} \mathrm{T}$ cell population as those of the control or PNT groups. Moreover, the inhibitory molecules, CTLA- 4 and TGF- $\beta$, are crucial to Treg suppressive functions. TGF- $\beta$ is required in the development of both natural and induced Tregs of thymic or peripheral origins (37). In this study, both the L-S-PNT and H-S-PNT groups had Tregs which highly expressed TGF- $\beta$ mRNA; the H-S-PNT group exhibited significantly $(\mathrm{P}<0.05)$ higher levels than the control group (Fig. 6B). Although FoxP3 gene expression did not differ among the groups, the expression of CTLA-4, which is known to be constitutively expressed in Tregs, was significantly upregulated in the aged ICR mice fed either PNT $(\mathrm{P}<0.05)$, L-S-PNT $(\mathrm{P}<0.001)$ or H-S-PNT $(\mathrm{P}<0.001)$. Since the stilbenes were removed in the PNT group, some minor factors may exist in peanut sprouts that may increase CTLA- 4 expression. Tregs may interact with CD80 or CD86 in dendritic cells in a CTLA-4 dependent manner, which renders indoleadmine 2, 3-dioxygenase production in dendritic cells to induce the catabolism of tryptophan into pro-apoptotic metabolites, resulting in 

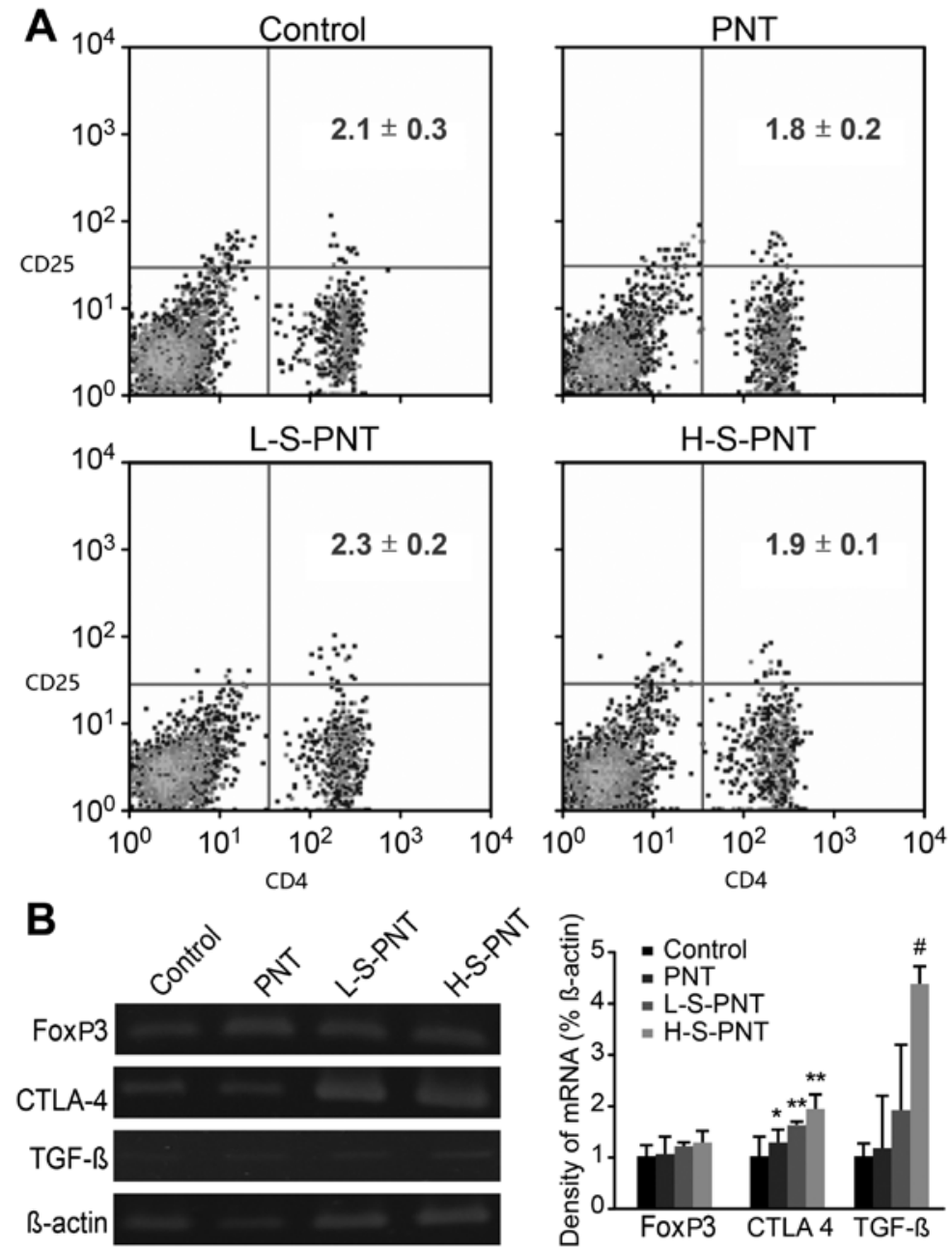

Figure 6. Mice fed with different levels of stilbenes rich peanut sprouts fortified diets for 48 weeks had no significant difference $\mathrm{CD} 4{ }^{+} \mathrm{CD} 25^{+} \mathrm{T}$ cell subset of pan $\mathrm{CD}^{+} \mathrm{T}$ cells population of spleno-lymphocytes (A). $\mathrm{CD} 4^{+} \mathrm{CD} 25^{+}$Tregs enriched with magnetic beads sorting kit were analyzed on gene expressions of Forkhead box P3 (FoxP3), CTLA-4, transforming growth factor- $\beta$ (TGF- $\beta$ ) and data were semi-quantitatively analyzed as shown in bar graph (B). Mice with free access to the diet supplemented with stilbenes rich peanut sprouts exhibited a dose-dependent increase in CTLA-4 and TGF- $\beta$ gene expression in Tregs. Significant difference in comparison with the control at ${ }^{*, *} \mathrm{P}<0.05$, and was at ${ }^{* * *} \mathrm{P}<0.001$; data were expressed as mean $\pm \mathrm{SD} ; \mathrm{n}=3$.

T effector cell apoptosis (38). Baeza et al (39) suggested that estrogen or phytoestrogen treatments are beneficial to aging or overiectomzied rats by affecting lymphocyte proliferation and NK cell activity. Immunological benefits to aging individuals were not associated with Treg populations, but emphasized their antioxidant abilities. Recently, Wang et al (40) reported that the peripheral blood Treg number and CTLA-4 gene expression were increased in mice with obesity due to a highfat diet by Res supplementation. In this study, in our ex vivo experiments, Ara-1 was shown to be much more potent than Res in promoting IL-10 and CTLA-4 expression, but not TGF- $\beta$. Unlike immune organs, the spleen and thymus have a higher ER $\beta$ mRNA expression than ER $\alpha$ (28), whereas ER $\alpha$ is mainly expressed in CD4 and CD8 T cells (29). In the present study, due to thymus involution in aging mice, thymic samples were not sufficient for analysis, which also indirectly explained why FoxP3 gene expression was low and there was no difference in FoxP3 expression among the treatment groups. Dietary peanut sprout powder rich in stilbenes exerted potent effects on peripheral induced-Treg functions in aged mice, and the upregulation of CTLA- 4 and TGF- $\beta$ in Tregs is essential for controling chronic inflammation.
In conclusion, the data demonstrate that Treg-mediated immunosuppression in aged individuals is crucial in managing increased pro-inflammatory conditions resulting from higher ratios of memory $\mathrm{T}$ cell and effector $\mathrm{T}$ cell activities reacting to excessive autoantigens in aged individuals. Res of the stilbene family has been reported to extend lifespan in several species, particularly in mice fed a high-calorie diet (41). Although some controversial aspects of the effects of Res on lifespan were raised against dietary calorie-restriction in the elderly $(40,42)$ the results of the present study provided an alternative mechanism by its potent phytoestrogenic activity on Treg cell activity. Upregulated Tregs are dominant in centenarians and are associated with longevity. Arachidin-1 is abundantly found in peanut sprouts and has a chemical structure similar to tht of Res, but with an additional hydroxyl and isopentenyl groups. It exhibited more potent phytoestrogenic property in the modulation Treg functions than Res, as demonstrated in this study. Thus, Res and Ara-1 may be beneficial in successful aging and longevity through their phytoestrogenic activity. They are potent in modulating regulatory $\mathrm{T}$ cell inhibitory functions by upregulating CTLA- 4 , TGF- $\beta$ and IL-10 expression. 


\section{Acknowledgements}

The present study was supported by the NSC 97-2321-B-415-00 grant, the Ministry of Science and Technology (former name National Science Council), Taiwan, R.O.C.

\section{References}

1. Gregg R, Smith CM, Clark FJ, Dunnion D, Khan N, Chakraverty R, Nayak L and Moss PA: The number of human peripheral blood $\mathrm{CD} 4{ }^{+} \mathrm{CD} 25^{\text {high }}$ regulatory $\mathrm{T}$ cells increases with age. Clin Exp Immunol 140: 540-546, 2005.

2. Zhao L, Sun L, Wang H, Ma H, Liu G and Zhao Y: Changes of $\mathrm{CD} 4^{+} \mathrm{CD} 25^{+} \mathrm{Foxp} 3^{+}$regulatory $\mathrm{T}$ cells in aged $\mathrm{Balb} / \mathrm{c}$ mice. J Leukoc Biol 81: 1386-1394, 2007.

3. Sharma S, Dominguez AL and Lustgarten J: High accumulation of T regulatory cells prevents the activation of immune responses in aged animals. J Immunol 177: 8348-8355, 2006.

4. Sakaguchi $\mathrm{S}$ : Naturally arising $\mathrm{CD}^{+}{ }^{+}$regulatory $\mathrm{T}$ cells for immunologic self-tolerance and negative control of immune responses. Annu Rev Immunol 22: 531-562, 2004.

5. Miyara $M$ and Sakaguchi $S$ : Natural regulatory $T$ cells Mechanisms of suppression. Trends Mol Med 13: 108-116, 2007.

6. Wan YY and Flavell RA: The roles for cytokines in the generation and maintenance of regulatory $\mathrm{T}$ cells. Immunol Rev 212: 114-130, 2006.

7. Dario AA: Vignali, Lauren W. Collision, and Creg J. Workman: How regulatory T cells work. Nat Rev Immunol 8: 523-532, 2008.

8. Hunt JS, Miller L, Roby KF, Huang J, Platt JS and DeBrot BL: Female steroid hormones regulate production of pro-inflammatory molecules in uterine leukocytes. J Reprod Immunol 35: 87-99, 1997.

9. Lengi AJ, Phillips RA, Karpuzoglu E and Ahmed SA: Estrogen selectively regulates chemokines in murine splenocytes. J Leukoc Biol 81: 1065-1074, 2007.

10. Pacifici R: Estrogen deficiency, T cells and bone loss. Cell Immunol 252: 68-80, 2008.

11. Cunningham $\mathrm{M}$ and Gilkeson $\mathrm{G}$ : Estrogen receptors in immunity and autoimmunity. Clin Rev Allergy Immunol 40: 66-73, 2011.

12. Bowers JL, Tyulmenkov VV, Jernigan SC and Klinge CM: Resveratrol acts as a mixed agonist/antagonist for estrogen receptors alpha and beta. Endocrinology 141: 3657-3667, 2000

13. Roupe KA, Remsberg CM, Yáñez JA and Davies NM: Pharmacometrics of stilbenes: Seguing towards the clinic. Curr Clin Pharmacol 1: 81-101, 2006.

14. Michel T, Halabalaki $M$ and Skaltsounis AL: New concepts, experimental approaches, and dereplication strategies for the discovery of novel phytoestrogens from natural sources. Planta Med 79: 514-532, 2013.

15. Chiou RY: Review: Resveratrol, a promising phytochemical in grapes, grape juices, wines and peanuts. Food Sci Agric Chem 4: 8-14, 2002.

16. Wotton HR and Strange RN: Increased susceptibility and reduced phytoalexin accumulation in drought-stressed peanut kernels challenged with Aspergillus flavus. Appl Environ Microbiol 53: 270-273, 1987.

17. Chang JC, Lai YH, Djoko B, Wu PL, Liu CD, Liu YW and Chiou RY: Biosynthesis enhancement and antioxidant and anti-inflammatory activities of peanut (Arachis hypogaea L.) arachidin-1, arachidin-3, and isopentadienylresveratrol. J Agric Food Chem 54: 10281-10287, 2006.

18. Gehm BD, McAndrews JM, Chien PY and Jameson JL: Resveratrol, a polyphenolic compound found in grapes and wine, is an agonist for the estrogen receptor. Proc Natl Acad Sci USA 94: 14138-14143, 1997.

19. Pozo-Guisado E, Lorenzo-Benayas MJ and FernándezSalguero PM: Resveratrol modulates the phosphoinositide 3-kinase pathway through an estrogen receptor alpha-dependent mechanism: Relevance in cell proliferation. Int J Cancer 109: 167-173, 2004.

20. Djoko B, Chiou RYY, Shee JJ and Liu YW: Characterization of immunological activities of peanut stilbenoids, arachidin-1, piceatannol, and resveratrol on lipopolysaccharide-induced inflammation of RAW 264.7 macrophages. J Agric Food Chem 55: 2376-2383, 2007.

21. Gao X, Xu YX, Janakiraman N, Chapman RA and Gautam SC: Immunomodulatory activity of resveratrol: Suppression of lymphocyte proliferation, development of cell-mediated cytotoxicity, and cytokine production. Biochem Pharmacol 62: 1299-1308, 2001.
22. Weng BB, Lin YC, Hu CW, Kao MY, Wang SH, Lo DY, Lai TY, Kan LS and Chiou RYY: Toxicological and immunomodulatory assessments of botryosphaeran ( $\beta$-glucan) produced by Botryosphaeria rhodina RCYU 30101. Food Chem Toxicol 49: 910-916, 2011.

23. Lin BS, Lien TF, Chao MR, Lai YH, Chang JC, Chou SJ, Liao HF and Chiou RYY: Toxicological and nutraceutical assessments of peanut sprouts as daily supplments to feed Sprague-Dawley rats for 18 weeks. J Sci Food Agric 88: 2201-2207, 2008.

24. Huang CP, Au LC, Chiou RYY, Chung PC, Chen SY, Tang WC, Chang CL, Fang WH and Lin SB: Arachidin-1, a peanut stilbenoid, induces programmed cell death in human leukemia HL-60 cells. J Agric Food Chem 58: 12123-12129, 2010.

25. Pfeifer RW and Patterson RM: Modulation of lectin-stimulated lymphocyte agglutination and mitogenesis by estrogen metabolites: Effects on early events of lymphocyte activation. Arch Toxicol 58: 157-164, 1986.

26. Brown N, Nagarkatti $M$ and Nagarkatti PS: Diethylstilbestrol alters positive and negative selection of $\mathrm{T}$ cells in the thymus and modulates T-cell repertoire in the periphery. Toxicol Appl Pharmacol 212: 119-126, 2006.

27. Shiau AK, Barstad D, Loria PM, Cheng L, Kushner PJ, Agard DA and Greene GL: The structural basis of estrogen receptor/ coactivator recognition and the antagonism of this interaction by tamoxifen. Cell 95: 927-937, 1998.

28. Brandenberger AW, Tee MK, Lee JY, Chao V and Jaffe RB: Tissue distribution of estrogen receptors alpha (ER-alpha) and beta (ER-beta) mRNA in the midgestational human fetus. J Clin Endocrinol Metab 82: 3509-3512, 1997.

29. Adori M, Kiss E, Barad Z, Barabás K, Kiszely E, Schneider A, Kövesdi D, Sziksz E, Abrahám IM, Matkó J, et al: Estrogen augments the T cell-dependent but not the T-independent immune response. Cell Mol Life Sci 67: 1661-1674, 2010.

30. Arruvito L, Sanz M, Banham AH and Fainboim L: Expansion of $\mathrm{CD} 4{ }^{+} \mathrm{CD} 25^{+}$and $\mathrm{FOXP}^{+}$regulatory T cells during the follicular phase of the menstrual cycle: Implications for human reproduction. J Immunol 178: 2572-2578, 2007.

31. Walker LS: CD $4^{+}$CD $25^{+}$Treg: Divide and rule? Immunology 111: 129-137, 2004.

32. Matejuk A, Dwyer J, Zamora A, Vandenbark AA and Offner H: Evaluation of the effects of 17 beta-estradiol (17beta-e2) on gene expression in experimental autoimmune encephalomyelitis using DNA microarray. Endocrinology 143: 313-319, 2002.

33. Mjösberg J, Svensson J, Johansson E, Hellström L, Casas R, Jenmalm MC, Boij R, Matthiesen L, Jönsson JI, Berg G and Ernerudh J: Systemic reduction of functionally suppressive CD $4{ }^{\text {dim }} \mathrm{CD} 25^{\text {high }}$ Foxp $3^{+}$Tregs in human second trimester pregnancy is induced by progesterone and 17 beta-estradiol. J Immunol 183: 759-769, 2009.

34. Sharma S, Chopra K, Kulkarni SK and Agrewala JN: Resveratrol and curcumin suppress immune response through CD28/CTLA-4 and CD80 co-stimulatory pathway. Clin Exp Immunol 147: 155-163, 2007.

35. Polanczyk MJ, Carson BD, Subramanian S, Afentoulis M, Vandenbark AA, Ziegler SF and Offner H: Cutting edge: Estrogen drives expansion of the $\mathrm{CD} 4{ }^{+} \mathrm{CD} 25^{+}$regulatory $\mathrm{T}$ cell compartment. J Immunol 173: 2227-2230, 2004.

36. Tai P, Wang J, Jin H, Song X, Yan J, Kang Y, Zhao L, An X, Du X, Chen X, et al: Induction of regulatory $\mathrm{T}$ cells by physiological level estrogen. J Cell Physiol 214: 456-464, 2008.

37. Vignali DAA, Collison LW and Workman CJ: How regulatory T cells work. Nat Rev Immunol 8: 523-532, 2008.

38. Mellor AL and Munn DH: IDO expression by dendritic cells: Tolerance and tryptophan catabolism. Nat Rev Immunol 4: 762-774, 2004.

39. Baeza I, Alvarado C, Alvarez P, Salazar V, Castillo C Ariznavarreta C, Fdez-Tresguerres JA and De la Fuente M: Improvement of leucocyte functions in ovariectomised aged rats after treatment with growth hormone, melatonin, oestrogens or phyto-oestrogens. J Reprod Immunol 80: 70-79, 2009.

40. Wang B, Sun J, Li X, Zhou Q, Bai J, Shi Y and Le G: Resveratrol prevents suppression of regulatory $\mathrm{T}$-cell production, oxidative stress, and inflammation of mice prone or resistant to high-fat diet-induced obesity. Nutr Res 33: 971-981, 2013.

41. Baur JA, Pearson KJ, Price NL, Jamieson HA, Lerin C, Kalra A, Prabhu VV, Allard JS, Lopez-Lluch G, Lewis K, et al: Resveratrol improves health and survival of mice on a high-calorie diet. Nature 444: 337-342, 2006.

42. Austad S: Recent advances in vertebrate aging research 2009. Aging Cell 9: 297-303, 2010. 